\title{
Téoros
}

Revue de recherche en tourisme

\section{Romantisme, féerie, fête et jeu : les origines des parcs d'amusement thématiques}

\section{Extraits de la thèse de doctorat d'Yves Robillard}

\section{Marie-Janou Lusignan}

Volume 12, numéro 3, octobre 1993

Le renouveau des parcs à thèmes

URI : https://id.erudit.org/iderudit/1077929ar

DOI : https://doi.org/10.7202/1077929ar

Aller au sommaire du numéro

Éditeur(s)

Université du Québec à Montréal

ISSN

0712-8657 (imprimé)

1923-2705 (numérique)

Découvrir la revue

Citer cet article

Lusignan, M.-J. (1993). Romantisme, féerie, fête et jeu : les origines des parcs d'amusement thématiques : extraits de la thèse de doctorat d'Yves Robillard. Téoros, 12(3), 3-4. https://doi.org/10.7202/1077929ar d'utilisation que vous pouvez consulter en ligne. 


\section{Romantisme, féerie, fête et jeu : les origines des parcs $d^{\prime}$ amusement thématiques Extraits de la thèse de doctorat d'Yves Robillard Marie-Janou Lusignan"}

Les parcs à thèmes sont indéniablementunecomposante majeure de l'industrie touristque contemporaine. Ces parcs sont le fruit d'un long processus qui, grâce à des influences diverses, a modelé leur caractère et leur personnalité. Ils ont évolué au gré des modes et des exigences dont les plus fondamentales sont la fete et le jeu. Monsieur Yves Robillard s'est penché il y a quelques années sur l'historique des parcs d'amusement. Il a soutenu une thèse de doctoraten 1983 intitulée L'esthétique des parcs d'amusement: justification théorique d'un «fun palace $*$, sous la direction de Gilbert Lascault de l'Universitéde Paris $X$. Une partie de cette thèse s'attarde aux origines des parcs d'attractions tels que connus aujourd'hui et leurs sources d'influence ${ }^{(1)}$.

\section{Parc à thèmes ou parc d'attraction? L'ímbroglio des définitions}

Il arrive souvent d'utiliser indifféremment les termes de parc d'amusement, parc d'attractions, luna-park ou fete foraine pour parler des parcs à thèmes. Il importe cependant d'apporter des distinctions à ces différentes expressions. Tel que l'explique monsieur Robillard:

Le terme ofete foraines veut dire en France un ensemble de macbines à vertige et de kiasques pour divers jeux et spectades que lion installe montentanément sur une place publique. Une afete foraines dure rarementplus d'unesemaine. Dans La province de Québec, nous appelons ces fetes des *expositions régionales", car elles ont toujours lieu à lexposition annuelle des produits dune région. Aux Etats-Unis, on Les appellera *fair (foire) si elles ont lieu dans le cadre d'une exposition régionale, ou «arnavals, si elles en sont indépendantes ou pour ne parler que des jeux. Les «fetes forainess

Madame Marievanou Lusignan termine sa maitrise en musbologie a I'UOAM et est responsable des Chroniques de Téros. contemporaines sont la continuation des anciennes foires d'antan, dont lorigine remonte au moyen-âge et même avant. Le parcdamusenent est une autre réalité. Il est permanent. II vient des apleasure gardenso (jardins de plaisance) anglais du XVIII siede. II doit beaucoup de ser traits à la foire, mais également aussi aux expositions universelles et internationales $d u$ $X T X^{\bar{r}}$ sièle.

La fete foraine se différencie donc, par ses caractéristiques, des pares d'amusement, d'attractions ou luna-park qui sont en fait des termes synonymes. Cependant, il convient mieux, selon monsieur Robillard, d'utiliser l'expression sparcd'amusementw car elle traduit sans ambiguïté le terme *amusementparbsaméricain et luiconfere un sens plus dynamique que le mot *attractions qui réfêre davantage à une participation passive des visiteurs.

Quant au «parc à thèmess ou «parc thématiques, une définition a été donnée par la compagnie américaine de pares d'amusement Six Flags Inc. en 1973 dans le document intitulé Analyse de la possibilité d'ouverture d'un nouveau parc de loisirs à thèmes dans la ville de Paris. Selon cet organisme:

Un parc à tbènes est un parc ou cbaque détail, tant du concept que de l'exploitation, a pour but de contribuer à créer wne atmosphère d'anusement et de rêve... Chaque partic du parc, du snack-bar aux attractions, est construite selon un modèle bistorique ou imaginaire, ce dernier élément étant surtout tiré d'bistoires populaires... Catte apparence de rôve est maintenue à travers le parc et, par exemple, une attraction n'est jamais que cela, car les vébicules et les décors ont un tbìme qui gjoute toujours une dimension imaginaires. Cest avec la création de Disneyland que lexpression oparc à tbèmess s'est surtout popularisée.

\section{Des racines européennes}

Les origines les plus lointaines du parc d'amusement semblent se trouver au moyen-âge (XIV`a au XVI siècle) à Hesdin, dansle nord dela France. Dansledomaine du duc de Bourgogne, des animaux sauvages se promenaient en liberté dans le parc où se trouvaitégalement diverses attractions tels qu'un labyrinthe, un immense cadran solaire, des automates, et également une *galerie des engins\%, sorte de maison des horreursoù lesvisiteurs se faisaient arroser, enfariner, poursuivre, battre et persécuter par différents engins!

Mais le parc d'amusement contemporain tire essentiellement ses origines de deux divertissements forts courus par les Européens au cours des derniers siècles: la foire et les «pleasure gardens londoniens du XVIII ${ }^{c}$ siècle. D'ailleurs, l'américain William F.Mangels, inventeur deplusieurs machines à vertiges au début du siècle, écrivit dans un livre intitulé The Outdoor Amusement Industry (1952) que:
Le parc d'amusement moderne américain a une bistoire relati- vewent courte, mais durant plus de trois cents ans ont existé dans les pays européens des contres élaborés d'amusement de plein air. Connus en général sous le nom de opleasure gardenso (jardins de plaisance ou de plaisir), ils étaient remarqua- blement semblables à nos parcs d'amusement actuels tant au point de vue de la conception d'ensemble que de la variété des divertisoments offerts.

Jusqu'au premier quart du $\mathrm{XX}^{\epsilon}$ siềcle, les activités des parcs d'amusement ressemblaient énormément à ceuxdu XIX ${ }^{e}$ siècle:

Tout était centré autour du kiosque
à musique, du concert. Il y avait
toujours un restaurant, wne salle de
bal ou une piste de danse, et un
tbéatre ou autre lieu de spectade.
Ony donnaitrégulièrement desfêtes
et mascarades. II y avait towjours 
une grande attraction de saisan [...] pour attirer les foules: acrobates, funambules ou artistes de renom. La soirée se terminait par un feu d'artifices. L'illunination du parc était très importante: tout devait concourir à créer une ambiance féerique. Y abondait les pavillons exotiques et autres décors pittoresques. Un droit d'entrée était exigible.

\section{L'ambiance des foires}

Au cours du XVIII" siècle, Londres et Paris se firent l'hôte de nombreuses foires. Plusieurs petits commerçants y prenaientplace en plus des restaurants et des cafés, mais les plus importants divertissements se manifestaient dans les nombreux spectacles de curiosités humaines ou animales, les animaux sauvages, les jongleurs et les magiciens, les figures de cires, les cabinets de physique, les automates, les acrobates et les funambules, les chanteurs et les musiciens, les danseurs, etc, mais aussi les pièces de thêatre (marionnettes ou acteurs vivants) dont la popularité vidait les salles de thếâtres protégées. On y jouait surtout des parodies, des opéras-comiques et des pièces du répertoire de la Comedia del Arte.

\section{C'està la foire qu'on est alléchercher au XIX' siècle la majorité des spectacles destinés à amuser le public [...] L'envirannement de la foire et les divers spectacles auxquels elles donnaient lieu, créaient une ambiance de festivités où prévalait le sentiment du merveilleux. Le parc d'amusement doit beaucoup à l'esprit de festivités des foires et à leur sens du *merveilleuxw.}

Construit en 1766, le Prater de Vienne est leplus ancien parcd'amusement au monde. Il a toujours été considérécomme une foire permanente. Selon monsieur Robillard, le Prater constitue une expérience unique au monde *que seul Disney World a égalé de nos jours».

\section{La magie des jardins de plaisance}

Contrairement à la foire, qui était une distraction populaire, le pleasure garden (jardin de plaisance) s'adressait davantage aux classes aisées. Parmi ceux qui ont le plus influencé les parcs d'amusement mo- derne, citons le Vauxhall(t), le plus ancien (1661)etleplusimportant, la Ranelegah, le Marylebone et le Cuper. Ils ont indubitablementservisdemodèles aux parcs d'amusement subséquents.

C'est au Vauxhall qu'on organisa pour la première fois des bals masqués en plein air, activité qui dérive en quelque sorte du thêâtre, un art qui influença grandement les «pleasure gardens»du XVIII ${ }^{\star}$ siècle. Les jardins devenaient ainsi «unlieu permanent de fête au décor enchanteurs.

On trouvait au Vauxhall plusieurs sentiers agrémentés de nombreux éléments de décoration, un orchestre, des pavillons, des cabinets à dîner, des spectacles, des concerts. Comme en témoigne monsieur Robillard, *certains soirs, la fête consistait uniquement à se promener dans le décor enchanteur, à y écouter de la musique et à y manger en public, mais d'autres soirs, c'était l'apogée de la fêtre avec les bals et mille autres fantaisiess. Habituellement, la soirée était couronnée par un feu d'artifice.

À l'instar des jardins de plaisance londoniens, Paris se dota elle aussi de jardins semblables, où les valeurs de la fete, de la magie, du thễutre et du jeu prenaient place.

\section{L'imaginaire des Expositions Universelles}

Les Expositions Universelles, elles aussi, influencèrent grandement les parcs d'amusement du XIX' siècle. C'est à l'Exposition Universelle de Chicago, en 1893 , que l'on retrouve pour la première fois une zone d'amusement. Elle avait été ajoutée après six mois d'opération afin d'attirer un plus grand nombre de visiteurs. *LeMidway Plaisance, commeon appelait cette zone, devint pour les Américains un symbole, un modèle, la colonne vertébrale des futurs parcs d'amusement». Une esquisse de cette zone d'amusement se retrouvait dans des Expositions Universelles antérieures, notamment à Paris en 1878 avec la *Rue des Nations» et en 1889 avec la *Rue du Caire», à Vienne en 1873 avec ses édifices pittoresques habités par des gens en costume national, etc. Lors de la première Fxposition Universelle à Londres, en 1851, aucune zone d'amusement n'était intégrée. Cependant, un peu partout dans la villedes établissementsoffraient des divertissements et des spectacles. C'est ainsi que l'on comprit rapidement *qu'en plus de l'étalage des produits du génie de chaque pays, il fallait offrir l'équivalent des anciennes foires et «pleasure gardens $»$ du XVIII' siècle, un monde fictif où en participant activement, le public imaginerait qu'il refait à sa façon l'histoire du monde*. Enconclusion, Yves Robillard affirme que:

Le parc d'amusement doit à la foire son esprit gouailleur et populacier. ses jeux de basard, d'babileté et de vertige, ses boutiques de bibloteries et de clinquant, ses spectacles populaires, que ce soit son thêâtre de vaudeville et d'opérettes, ses phénomènes de la nature, acrobates, monstres, animaux raresou savants, spectacles de vulgarisation scientifique ou ses emironnements exotiques, musées de cire, maisons de l'illusion (spectacles mécaniques d'architecture), enfin son sens du merveilleux manifesté par tous ces moyens.

Le parc d'amusement doit aux pleasure gardens ses concerts, ses bals, ses repas en public, l'esprit de la fete baroque transporté des grandes cours européennes dans un parc public offrant un enviromnement unifié avec sès décors de rêve, ses salles d'apparat, sesjardins fabuleux, son esprit théâtral, ses mascarades, ses illuminations, ses feux d'artifices et un style décoratif souvent bérité du rococo.

C'est ainsi que la fête, l'un des besoins les plus fondamentaux de l'humain, trouve en grande partie réponse à ses exigences dans les parcs à thèmes. $f$

\section{Notes}

(1) Monsieur Yves Robillard a également fait paratté dans la revue Mudiart, en octobre 1972, une histoire chronologique des manèges et des parcs d'attractions dans le monde ainsi qu'un article intitule Considérations générales sur le parc d'attraction décrivant et expliquant les principales caractéristiques de ces lieux. II à également été co-auteur d'un article portant sur l'idée d'un fun palace dans la revue Madiart, mars 1972.

(2) NDLR: Montréal se dota togalement à la fin du XVIII" sibcle d'un jardin de plaisance que le promoteur nommait prétentieusement le Vauxhall d'Amerique. Malheureusement, il n'eut qu'un succés de courte durée mais l'idée fut reprise au début du XIX sibcle avec les Jardins Guilbault et le Parc Sohmer. 